\title{
Charge-Remote Fragmentation in a Hybrid (BEqQ) Mass Spectrometer To Determine Isotopic Purity in Selectively Polydeuterated Surfactants
}

\author{
Albert A. Tuinman, Kelsey D. Cook, and Linda J. Magid \\ Department of Chemistry, University of Tennessee, Knoxville, Tennessee, USA
}

The combination of fast atom bombardment with charge-remote fragmentation using a hybrid (BEqQ) mass spectrometer was used successfully to assess and localize the extent of selective deuterium isotope labeling of tetradecyltrimethylammonium bromides. Spectral details reveal a new reaction that can give rise to ions isobaric with those formed by chargeremote fragmentation. (J Am Soc Mass Spectrom 1990, 1, 85-91)

(1) ne of the first applications of mass spectrometry, and still a very important one, is the identification of isotopes; in fact, Thomson and Aston are generally credited with the "discovery" (or at least the confirmation) of neutrons in their earliest mass spectrometry experiments. More recent applications include the broad field of stable isotope labeling, which is used to discern mechanistic details and/or kinetic isotope effects in such areas as bioorganic and physical organic chemistry, and materials labeling (e.g., in metabolic pharmacology and forensics). In such applications it is frequently important to Iocalize an isotope label at a specific site in the molecule as well as to determine the overall extent of labeling.

One such application derives from structural studies of colloidal aggregates in solution. Using techniques such as small-angle neutron scattering (SANS) or two-dimensional nuclear magnetic resonance (NMR) for surfactant micelles and lipid bilayers formed from selectively labeled monomers, one can obtain information on the distribution of distances between labeled groups in the aggregates and hence on hydrocarbon tail organization within the aggregates' cores. For both SANS and NMR, the preferred labeling schemes involve one or two protiated sites in an otherwise perdeuterated molecule. Clearly, interpretation of the scattering pattern relies on accurate knowledge of the site(s) and the extent of labeling.

In the course of preparing surfactants for use in SANS and two-dimensional NMR studies of micelles, two highly deuterated analogues of tetradecyltrimethylammonium bromide $\left(1\right.$, nominally $\left.\mathrm{D}_{0}^{+} \mathrm{Br}^{-}\right)$ were prepared. In the first, the long alkyl chain is to-

Address reprint requests to Albert A. Tuinman, Department of Chemistry, University of Tennessee, Knoxville, IN 37996-1600. tally deuterated (2, nominally $\mathrm{D}_{29}^{+} \mathrm{Br}^{-}$). The second is labeled at the $N$-methyls as well as at all but the $\omega$ carbon of the chain $\left(3\right.$, nominally $\left.\mathrm{D}_{35}^{+} \mathrm{Br}^{-}\right)$. Note that the isotopically "pure" ions are $\mathrm{D}_{0}^{+}, \mathrm{D}_{29}^{+}$, and $\mathrm{D}_{35}^{+}$(the counterion is $\mathrm{Br}^{-}$in each case); 1,2 , and 3 designate the isotopically impure bulk samples.

$$
\begin{array}{lll}
1 & \left(\mathrm{CH}_{3}\right)_{3} \mathrm{~N}^{+}\left(\mathrm{CH}_{2}\right)_{13} \mathrm{CH}_{3} & \left(=\mathrm{D}_{0}^{+}\right) \mathrm{Br}^{-} \\
2 & \left(\mathrm{CH}_{3}\right)_{3} \mathrm{~N}^{+}\left(\mathrm{CD}_{2}\right)_{13} \mathrm{CD}_{3} & \left(=\mathrm{D}_{29}^{+}\right) \mathrm{Br}^{-} \\
3 & \left(\mathrm{CD}_{3}\right)_{3} \mathrm{~N}^{+}\left(\mathrm{CD}_{2}\right)_{13} \mathrm{CH}_{3} & \left(=\mathrm{D}_{35}^{+}\right) \mathrm{Br}^{-}
\end{array}
$$

Because of its high sensitivity to precharged surfactants [1], fast atom bombardment (FAB) should provide a means of efficiently sampling these analytes. Although simple abundance measurements in the molecular ion region (i.e., near the mass of the preformed surfactant cation) could optimally be expected to indicate the degree of labeling of 2 and 3 , the FAB spectrum will not generally provide sufficient information about the positions at which labeling (or mislabeling) has occurred.

Charge-remote fragmentation (CRF) has proved to be particularly versatile in determining structural features of long alkyl chains [2]. The positions of hydroxy groups [3, 4], double bonds [5-7], and sites of branching $[3,4,8]$ have been localized. Significantly, this fragmentation has been shown [9] to proceed without isotope label scrambling, making it ideally suited for labeling studies.

We assess now whether FAB/MS in conjunction with CRF can be used to determine the extent and localization of any mislabeling of the surfactant cations $\mathrm{D}_{29}^{+}$and $\mathrm{D}_{35}^{+}$. 


\section{Experimental}

The surfactants 2 and 3 were synthesized [10] from the appropriately labeled 1-bromotetradecane supplied by MSD Isotopes (Pointe Claire-Dorval, Quebec, Canada). Purity was assessed by elemental analyses and by comparison of the surfactants' critical micelle concentrations (established using a $\mathrm{Br}^{-}$ion selective electrode) with literature values [11].

Mass spectra were obtained on a hybrid mass spectrometer of $\mathrm{BEqQ}$ configuration, the $\mathrm{ZAB}-\mathrm{EQ}$ from $\mathrm{VG}$ Analytical (Manchester, England). In all cases, 8-keV xenon FAB was used with a glycerol matrix $(\sim 3 \mu \mathrm{g}$ analyte/mg glycerol). For "high-energy" (i.e., $8 \mathrm{keV}$, laboratory frame) collision-activated dissociation (CAD). helium was used as collision gas in the first $(B / E$ linked scan) or second (mass-analyzed ion kinetic energy spectroscopy, MIKES) field-free regions at such pressures as to reduce the precursor ion abundance by 50\%. "Low-energy" CAD (100-500 eV, laboratory frame) was carried out with Q-scans, that is, separation of a precursor ion with $\mathrm{BE}$, followed by deceleration, then muitiple collisions with argon at controlled kinetic energy in $q$, and analysis of the collision products with $Q$. The argon pressure measured with an ionization gauge outside $q$ was $1.5 \times 10^{-5}$ torr, representing $\sim 3$ mtorr in the 254-mm-long collision quadrupole. All product ion spectra were collected using VG's multichannel acyuisition mode (MCA), a software simulation of true multichannel acquisition. Spectra represent summation of at least 16 scans of $10 \mathrm{~s}$ each.

\section{Results and Discussion}

\section{Extent of Labeling}

The FAB mass spectra of 1,2 , and 3 are dominated by the intact, even-electron molecular ions $(m / z 256,285$. and 291 , respectively; hereafter designated $\mathrm{M}^{+}$). The regions around $\mathrm{M}^{+}$are presented in Figure 1 (resolution $\sim 25,000)$. Before ascribing ions at $[\mathrm{M}-1]^{+}$and $[\mathrm{M}-2]^{+}$in Figure $1 \mathrm{~b}$ and $\mathrm{c}$ to mislabeling of $\mathrm{D}_{29}^{+}$ and $\mathrm{D}_{35}^{+}$(incorporation of one $\mathrm{H}$ instead of $\mathrm{D}$ ), it is necessary to consider the evidence of bombardmentinduced hydrogen loss in Figure 1a. The ion at $[\mathrm{M}-2]^{+}$ in Figure 1a is almost certainly due to the loss of $\mathrm{H}_{2}$ from $\mathrm{D}_{0}^{+}$, since that reaction can be observed in the high-energy $C A D$ spectrum (see Figure $2 b$, below). This extent of $\mathrm{H}_{2}$ loss suggests two possible isobaric assigmments for the ion at $[\mathrm{M}-1]^{+}:[\mathrm{M}-\mathrm{H}]^{+}$or the ${ }^{13} \mathrm{C}$ component of $\left.\left[\mathrm{M}-\mathrm{H}_{2}\right]\right]^{+}$. At the resolution used for the spectrum of Figure $1 \mathrm{a}(\mathrm{m} / \Delta m=25,000)$, it is clear that only ${ }^{13} \mathrm{C}\left[\mathrm{M}-\mathrm{H}_{2}\right]^{+}$was present. Furthermore, the abundance of $[\mathrm{M}-1]^{+}$is $18 \%$ of $[\mathrm{M}-2]^{+}$, in close agreement with expectations for ${ }^{13} \mathrm{C}\left[\mathrm{M}-\mathrm{H}_{2}\right]^{+}(19 \%)$.

Having established that no single hydrogen (and therefore no deuterium) atoms are lost from the molecular ion in the FAB process, peaks at $[M-1]^{+}$for $D_{29}^{+}$ and $\mathrm{D}_{35}^{+}$can be confidently attributed to incomplete labeling (i.e., one too few $\mathrm{D}$ and one too many $\mathrm{H}$ ). For convenience we designate these singly mislabeled impurities $D_{28}^{+}$and $D_{34}^{+}$. The abundances of $D_{28}^{+}$and $D_{34}^{+}$

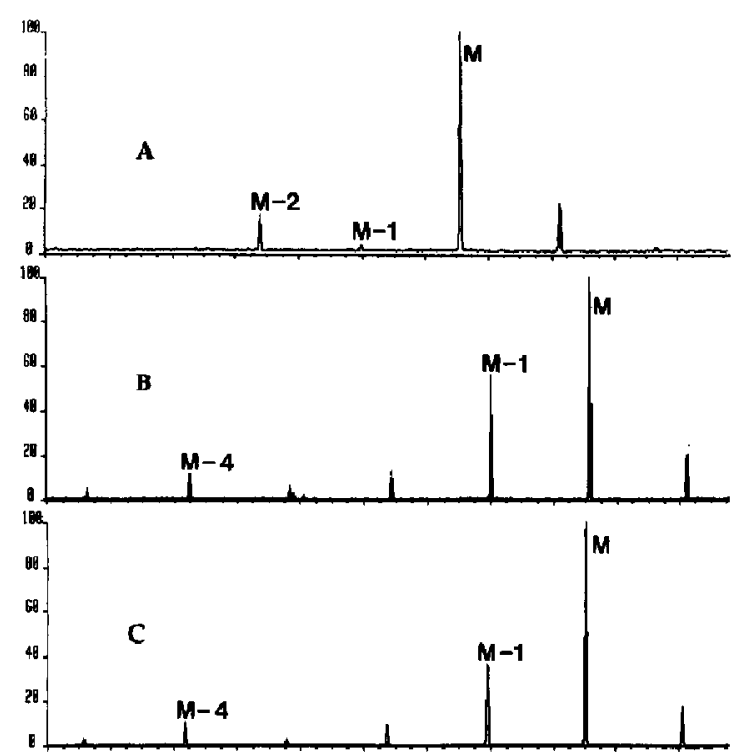

Figure 1. The molecular cation regions of 1,2 , and 3, recorded with 25,000 resolving power.

are $47.9 \%$ and $38.4 \%$, respectively, relative to the intensities at $1 \mathrm{u}$ higher mass. While this may seem like a high isotope impurity, it can be compared with the $19.6 \%$ "impurity" at $[\mathbf{M}+1]^{+}$of $\mathbf{1}$. The latter derives mainly from the $1.1 \%$ natural abundance of ${ }^{13} \mathrm{C}$. Each site is therefore $98.9 \%$ isotopically pure ${ }^{12} \mathrm{C}$. By analogy, each of the 29 supposedly deuterated sites of $\mathrm{D}_{29}^{+}$ is $98.2 \%$, and the sites of $\mathrm{D}_{35}^{+}$are $98.8 \%$ isotopically pure $\mathrm{D}$, assuming random distribution of the mislabeling (see below). [These values are corrected for the natural ${ }^{13} \mathrm{C}$ abundance contributions $\left(\mathrm{D}_{28}{ }^{13} \mathrm{C}_{1}\right.$ and $\left.\mathrm{D}_{34}{ }^{13} \mathrm{C}_{1}\right)$ to the nominal masses of the correctly labeled peaks $D_{29}$ and $D_{35}$.] Using these numbers, the expected doubly mislabeled abundances $\left(\mathrm{D}_{29}^{+}-2\right.$ and $\left.\mathrm{D}_{35}^{+}-2\right)$ are $10.0 \%$ and $7.0 \%$. This accords reasonably well with the observed values $\left(12.4 \%\right.$ and $9.2 \%$ ). Loss of $D_{2}$ (analogous to $\mathrm{H}_{2}$ loss from $\mathrm{D}_{0}^{+}$) accounts for the ions at $[\mathrm{M}-4]^{+}$ in Figure $1 b$ and $c$, which do not interfere with the mislabeled ions at $[\mathbf{M}-2]^{+}$.

Although the possibility of significant hydrogen atom loss in the FAB process was discounted by the high-resolution experiment described above, the possibility of an exchange reaction (hydrogen from the glycerol matrix substituting for deuterium in the analyte) has not been eliminated. If such a reaction does, in fact, take place, the amount of apparent mislabeling at $[\mathrm{M}-1]^{+}$would be inflated. The figures quoted above for isotope purity $(98.2 \%$ and $98.8 \%$ ) are therefore to be considered "worst-case" values; the analytes may be isotopically purer than indicated. This possibility could be tested by substituting $d_{8}$-glycerol and comparing the measured ratios. However, because the observed values were reasonable and acceptable, the expense of pursuing the $d_{8}$-glycerol experiments was deemed unnecessary. 


\section{Determining the Positions of Isotopic Impurities by Tandem Mass Spectrometry}

It is unknown a priori whether the observed total isotopic impurities $(47.9 \%$ for 2 and $38.4 \%$ for 3 ) are evenly distributed among all labeled sites of the molecules, or concentrated at some position(s) as a result of the chemical synthesis. The latter would give rise to much lower isotope purities at mislabeled positions, thus seriously complicating neutron scattering studies. Charge-remote fragmentation with tandem mass spectrometry (MS/MS) [2] presents a possible means to resolve this question, but certain features of the problem require careful consideration.

In general, MS/MS may be thought of as comprising three distinct stages: isolation of the desired precursor ion, dissociation of that ion (either metastably or collision-induced), and analysis of the fragments thus created. For the problem outlined above (i.e., identification of the sites of isotopic mislabeling), each of these three stages of the MS/MS experiment has requirements that must be met.

Stage 1. The precursor ion of interest is the mislabeled one (i.e., $\mathrm{D}_{28}^{+}$and $\mathrm{D}_{34}^{+}$for 2 and 3, respectively), which must be isolated in the first stage of the MS/MS experiment with unit mass resolution or better to distinguish it from the more abundant fully labeled main component $\left(D_{29}^{+}\right.$or $\left.D_{35}^{+}\right)$. This precludes simple $B / E$ linked scanning with $\mathrm{CAD}$ in the first field-free region of a double-focusing mass spectrometer. Precursor ion resolution in such an experiment is poor $[12,13]$ and may result in superposition of product ion spectra of adjacent precursors. Figure $2 \mathrm{a}$ illustrates the well-resolved product ions generated by a B/E linked scan from precursors in the molecular ion region of 1 . Note that each product ion cluster represents a copy of the relative intensities observed for the precursor ions in Figure 1a. Better first-stage separation is needed.

Stage 2. CAD of the precursor must take place in such a fashion as to create fragments of known structure that will distinguish the different positions in the alkyl chain. Additionally, the CAD mechanism must exclude the possibility of isotope label scrambling before or during fragmentation. Otherwise, the observed results will not necessarily reflect the initial position of the label.

Charge-remote fragmentation applies chiefly to organic ions containing at least one relatively long hy- drocarbon chain and having charge localized by a heteroatom. This description applies precisely to our target ions, $D_{28}^{+}$and $D_{34}^{+}$. In the reported [9] CRF mechanism, summarized in Scheme I, the localization of charge and substituents remains unaffected by the reaction. This accounts for the lack of isotope scrambling observed [9] in the fragments 6 when using labeled analytes 4 of known positional integrity.

These characteristics make CRF ideal for investigating labeled surfactants. To see why this is true, consider the following example. If the site of mislabeling is the same on all $\mathrm{D}_{28}^{+}$parent ions (say $j$ carbons away from the charge site), then each product ion (6) with $n<j-2$ would be represented by a single peak not containing the mislabeling. When $n=j-1$ in 4 , the mislabeled site is placed at the position from which a hydron is lost, and this may be either the hydrogen ot the deuterium. Two product ions of equal abundance (ignoring kinetic isotope effects) would result, representing the presence and absence of the incorrect labeling. For every other product ion chain length $(6$, $n=j-2$ and $n \geq j$ ), a single peak would arise, incorporating the H-atom "error." Thus, the position of a specifically mislabeled site can, in principle, be identified unambiguously.

It is also possible to predict the appearance of a CRF spectrum in the case where mislabeling is totally random. Here, the incorporation of the $\mathrm{H}$ "error" would be statistically controlled, so that the expected ratio of isotope peaks for any particular product ion will be the ratio of the number of relevant atoms lost in the fragmentation to the number retained on the charged species 6. Between the extremes of selective and random mislabeling, partial enrichment of the mislabeling at certain sites would result in biased peak ratios for each olefin chain length in 6 . The positions and degree of "enrichment" should be calculable from the extent of deviation from the predictions for random mislabeling.

Stage 3. The pairs of product ions that characterize the isotope positions will be separated by only $1 \mathbf{u}$. This is the mass difference of the isotopes ( $H$ and $D)$ we need to distinguish. Therefore, resolution of the product ion spectrum should be unit mass, or at least sufficient to quantify overlapping peaks with reasonable accuracy.

Charge-remote fragmentation experiments are normally conducted in conjunction with MIKES. However, analysis by MIKES provides broad peaks [9] that



Scheme I

$$
\mathrm{CH}_{3}-\left(\mathrm{CH}_{2}\right)_{\mathrm{m}}-\mathrm{CH}=\mathrm{CH}_{2}
$$

5

$$
\mathrm{CH}_{2}=\mathrm{CH}-\left(\mathrm{CH}_{2}\right)_{\mathrm{n}}-\mathrm{x}^{+}
$$



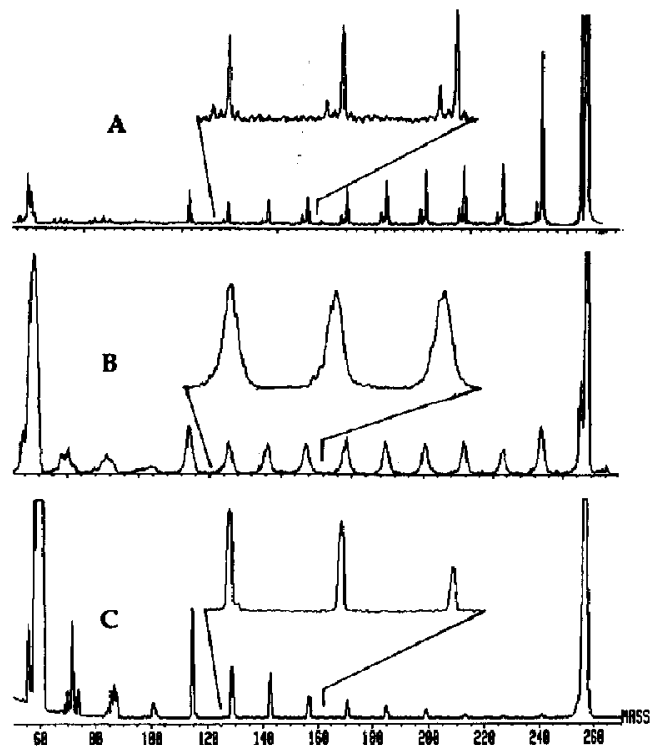

Figure 2. Product ion spectra resulting from argon CAD of the precursor $D_{0}^{+}$, using three different modes of analysis: (a) $R / E$ linked scan, (b) MIKES, and (c) Q-scan.

do not allow quantitation of masses separated by only 1 u. An example of the poor resolution is given in Figure 2b. Thus, MIKES is not suitable for this experiment.

Obviously what is needed is instrumentation that provides good resolution of both precursor and product ions while utilizing the relatively high collision energy best suited $[14,15]$ to achieving CRF. In other words, a four-sector instrument would seem ideal for this experiment. Such an instrument was used [9] to confirm the original determination of the positional integrity of labels with CRF. Because the feasibility of CRF has been demonstrated [16] in Fourier transform mass spectrometry, this represents another possibility, provided the instrument is equipped with a dual source allowing FAB-desorbed ions to be injected into the analyzer cell.

In lieu of such instruments, two somewhat more common (and less expensive) alternatives may be considered: the triple quad and the $\mathrm{BEqQ}$ hybrid. The first generally suffers from a limited energy range for $\mathrm{C} \triangle \mathrm{D}$ [17], often not exceeding $100 \mathrm{eV}$. Although it is occasionally possible to induce CRF at these energies (especially using a heavy target gas such as xenon or surface-induced dissociation), success appears to be compound-dependent, with less success for cations than for anions $[18,19]$. Charge-remote fragmentation was demonstrated [20] to work reasonably efficiently at $400 \mathrm{eV}$ on a $\mathrm{BEqQ}$ hybrid, which makes it a suitable compromise to fulfill the three criteria listed above.

Figure 2 presents the product ion spectra from the same precursor ion $\left(D_{0}^{+}\right)$in the three modes discussed: linked scan, MTKES, and hybrid Q-scan (see under Experimental). The altered envelope of product ions using the Q-scans has been noted previously and may represent an instrumental artifact discrimi-

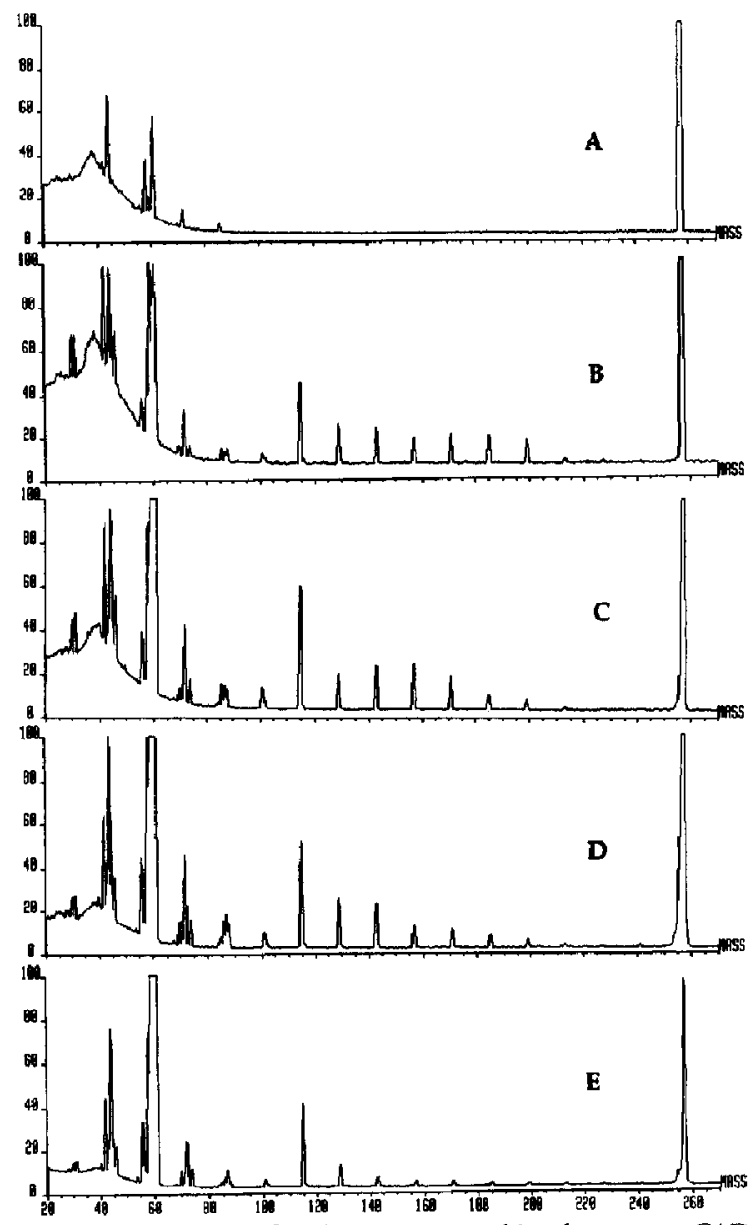

Figure 3. Q-scan product ion spectra resulting from argon CAD of the precursor $\mathrm{D}_{0}^{+}$at collision energies of (a) $100 \mathrm{eV}$, (b) 200 $\mathrm{eV}$, (c) $300 \mathrm{eV}$, (d) $400 \mathrm{eV}$, and (e) $500 \mathrm{eV}$.

nating against the upper mass range [18]. However, it does not affect the comparison of intensities at adjacent masses and therefore should not interfere with localization of mislabeling.

The inset in Figure $2 c$ shows a narrow part of the $Q$-scan and demonstrates the adequate resolution of both precursor and product ions by comparison with Figure $2 a$ and $b$. Q-scans were repeated at various collision energies (Figure 3) to establish the best parameters for the experiment. Spectra between 200 and 400$\mathrm{eV}$ (multicollision with argon) all seem adequate. The $400-\mathrm{eV}$ conditions were chosen for further experiments because there was less pronounced baseline distortion at low mass. [The high baseline at low mass observed in Figure 3a-c is due to high-energy ions (e.g., 400-eV precursor ions) that are not filtered by $Q$ when it is set at a low mass.]

To evaluate the proposed technique, a model analyte of known isotopic labeling (or mislabeling) is required. Such a substance is provided by the natural abundance of ${ }^{13} \mathrm{C}_{;}$we consider ${ }^{13} \mathrm{C}-\mathrm{D}_{0}^{+}$to be a mislabeled entity (with "isotope purity" of $98.9 \%$ for ${ }^{12} \mathrm{C}$ ). 


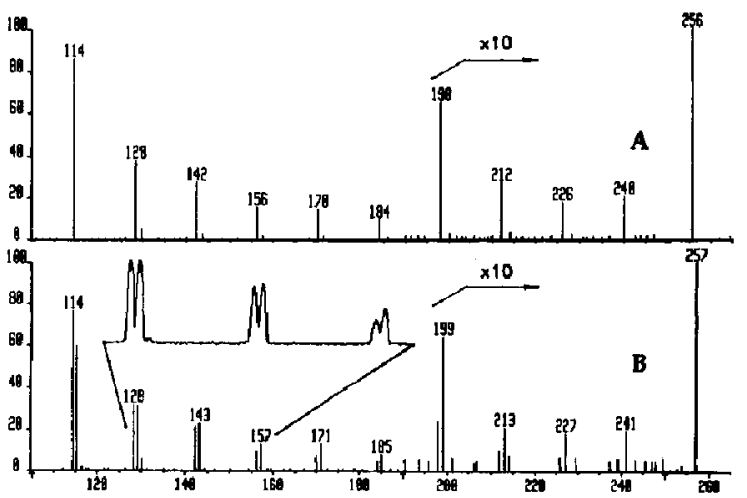

Figure 4. $400-\mathrm{eV} Q-s c a n$ product ion spectra of (a) isotopically pure $D_{0}^{+}$and (b) the peak at $D_{0}^{+}+1$, consisting mainly of ${ }^{13} \mathrm{C}-\mathrm{D}_{0}^{+}$. The inset shows an expanded part of the original (MCA) data from which the mass/intensity pairs were calculated.

The position of the "mislabeling" is evenly distributed among all carbons of the parent ion. The expected abundance ratio for pairs of product ions with a given number of carbons can thus be calculated (see above). Figure $4 \mathrm{a}$ and $\mathrm{b}$ shows the $\mathrm{Q}$-scan product ion spectra of $\mathrm{D}_{0}^{+}(\mathrm{m} / \mathrm{z} 256)$ and the "mislabeled" ${ }^{13} \mathrm{C}-\mathrm{D}_{0}^{+}(\mathrm{m} / \mathrm{z}$ 257). As expected, each CRF product ion in Figure $4 a$ is split into a pair of ions in Figure $4 b$, one retaining and one losing the ${ }^{13} \mathrm{C}$ "label."
Table 1 compares the expected abundance ratios of the product ion pairs with those found in Figure $4 \mathrm{~b}$. It can be seen that for product ion chain lengths from 4 to 8 , the agreement between observed and expected values is reasonably good (within $10 \%$ ). For the longer chain lengths, signal abundances become too weak (see Figure 4a) to establish the experimental ratios accurately, and the observed and expected values diverge radically.

Similarly, the observed product ion isotope ratios for $\mathrm{D}_{28}^{+}$and $\mathrm{D}_{34}^{+}$compare reasonably well with the calculated values (Table 1 and Figures 5 and 6), indicating that the distribution of the deuterium isotope mislabeling is also random. Deviations are somewhat greater for 3 than for 2, but there is no clear trend to indicate a nonrandom distribution.

The systematic trend of increasing negative deviations as product ion chain lengths increase (Table 1) deserves mention. This must reflect an instrumental artifact because it is evident even for product ions of $\left[D_{0}+1\right]^{+}$, which must have a random distribution of ${ }^{13} \mathrm{C}$. A likely explanation stems from the peak overlap evident in the insets to Figures 4-6 (MCA data), which will result in inflation of the measured intensity of the minor peak when the peak-detection algorithm is used to convert the MCA data to the mass/intensity format. The effect will be greatest when peak intensities differ most and absolute intensities are smallest.

Table 1. Ratios of product ion mass abundances for precursors that are singly mislabeled with an isotopic impurity

\begin{tabular}{|c|c|c|c|c|c|c|c|c|c|}
\hline \multirow{2}{*}{$\begin{array}{l}\text { Praduct } \\
\text { ion } \\
\text { chain } \\
\text { length }\end{array}$} & \multicolumn{3}{|c|}{$\begin{array}{c}\text { Precursor }=\left[\mathrm{D}_{0}+1\right]^{+} \\
(\mathrm{m} / \mathrm{z} 257)\end{array}$} & \multicolumn{3}{|c|}{$\begin{array}{l}\text { Precursor }=\mathrm{D}_{2 \mathrm{~B}}^{+} \\
(m / z 284)\end{array}$} & \multicolumn{3}{|c|}{$\begin{array}{l}\text { Precursor }=D_{34}^{+} \\
(m / z 290)^{4}\end{array}$} \\
\hline & $\begin{array}{l}\text { Product ion } \\
\text { Masses (u) }\end{array}$ & $\begin{array}{c}\text { Obs. } \\
\text { ratio }\end{array}$ & $\begin{array}{l}\text { Calc. } \\
\text { ratio }\end{array}$ & $\begin{array}{l}\text { Product ion } \\
\text { masses (u) }\end{array}$ & $\begin{array}{l}\text { Obs. } \\
\text { ratio } \\
\text { b,a }\end{array}$ & $\begin{array}{l}\text { Calc. } \\
\text { ratio }\end{array}$ & $\begin{array}{l}\text { Product ion } \\
\text { masses (u) }\end{array}$ & $\begin{array}{l}\text { Obs. } \\
\text { ratio } \\
\text { rad }\end{array}$ & $\begin{array}{l}\text { Calc. } \\
\text { ratio }\end{array}$ \\
\hline 4 & 115,114 & $\begin{array}{l}0.78 \\
(+7)\end{array}$ & 0.73 & 120,121 & $\begin{array}{l}0.33 \\
(+3)\end{array}$ & 0.32 & 129,130 & $\begin{array}{c}0.95 \\
(+13)\end{array}$ & 0.84 \\
\hline 5 & 129,128 & $\begin{array}{l}0.99 \\
(+6)\end{array}$ & 0.93 & 136,137 & $\begin{array}{l}0.48 \\
(+7)\end{array}$ & 0.45 & 145,146 & $\begin{array}{c}1.27 \\
(+20)\end{array}$ & 1.06 \\
\hline 6 & 143,142 & $\begin{array}{l}1.07 \\
(-9)\end{array}$ & 1.17 & 152,153 & $\begin{array}{l}0.63 \\
(+3)\end{array}$ & 0.61 & 161,162 & $\begin{array}{l}1.36 \\
(+2)\end{array}$ & 1.33 \\
\hline 7 & 157,156 & $\begin{array}{l}1.46 \\
(-1)\end{array}$ & 1.48 & 168,169 & $\begin{array}{l}0.78 \\
(-4)\end{array}$ & 0.81 & 177,178 & $\begin{array}{l}1.52 \\
(-1)\end{array}$ & 1.69 \\
\hline 8 & 171,170 & $\begin{array}{l}1.87 \\
(-1)\end{array}$ & 1.89 & 184,185 & $\begin{array}{c}0.93 \\
(\quad 13)\end{array}$ & 1.07 & 193,194 & $\begin{array}{l}1.61 \\
(\quad 26)\end{array}$ & 2.18 \\
\hline 9 & 185,184 & $\begin{array}{c}1.81 \\
(-25)\end{array}$ & 2.40 & 200,201 & $\begin{array}{l}1.17 \\
(-17)\end{array}$ & 1,41 & 209,210 & $\begin{array}{c}2.11 \\
(-27)\end{array}$ & 2.88 \\
\hline 10 & 199,198 & $\begin{array}{c}2.69 \\
(-17)\end{array}$ & 3.25 & 216,217 & $\begin{array}{c}1.53 \\
(-19)\end{array}$ & 1.90 & 225,226 & $\begin{array}{c}2.77 \\
\{-31\}\end{array}$ & 4.00 \\
\hline 11 & 213,212 & $\begin{array}{c}2.29 \\
(-51)\end{array}$ & 4.67 & 232,233 & $\begin{array}{c}2.02 \\
(-23)\end{array}$ & 2.62 & 241,242 & $\begin{array}{c}2.61 \\
(-57)\end{array}$ & 6.00 \\
\hline 12 & 227,226 & $\begin{array}{c}2.83 \\
(-62)\end{array}$ & 7.50 & 248,249 & - & 3.83 & 257,258 & - & 10.60 \\
\hline 13 & 240,241 & - & 16.00 & 264,265 & - & 6.25 & 273,274 & - & 30.84 \\
\hline
\end{tabular}

a Observed abundance ratio of the product ions containing the mislabel to those not containing the mislabel (i.e., high mass to low mass).

- Values in $(1$ indicate $\%$ deviation $=$ [iobs. - calc. $) / c a l c.] \times 100$.

c Calculated ratio high mass/low mass including the contribution to the high-mass product ion from ${ }^{15} \mathrm{~N}-\mathrm{D}_{0}^{+}$lan unresolved isobaric precursar comprising ca $2 \%$ of the signal at $\left[0_{0}+1\right]^{+1}$.

Observed abundance ratio of the product ions containing the mislabel to those not containing the mislabel (i.4., low mass to high mass).

a Calçloted low mass/high mass ratio. 


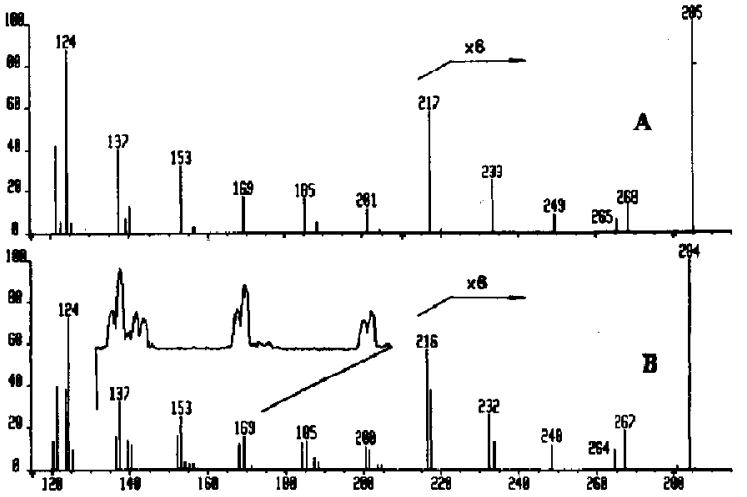

Figure 5. 400-eV Q-scan product ion spectra of (a) isotopically pure $\mathrm{D}_{29}^{+}$and (b) the mislabeled $\mathrm{D}_{28}^{+}$. The inset shows an expanded part of the original (MCA) data from which the mass/intensity pairs were calculated.

\section{A New Fragmentation Reaction}

Finally, Figure 5 displays a surprising anomaly: an ion series that parallels the CRF series at $3 u$ higher mass (i.e., at $m / z 124,140,156, \ldots$ in Figure $5 a$, as opposed to $121,137,153, \ldots$ for CRF). This series starts at greater abundance than CRF $\left(I_{124}>I_{121}\right)$ but diminishes more rapidly with increasing mass. As in CRF, each peak can be attributed to the nominal loss of an alkane from the precursor ion; in contrast to CRF, the anomalous loss includes one of the $\mathrm{N}$-methyls as well as atoms from the long alkyl chain. Loss of $N$-methyl hydrogens in lieu of alkyl deuteriums causes the 3-u shift. The heaviest member of this series (corresponding to loss of $\mathrm{CH}_{3} \mathrm{D}, \mathrm{m} / \mathrm{z} 268$ ) is surprisingly abundant but has precedence [21]. Its analogue for $D_{35}^{+}$is found at $m / z 271$ in Figure 6a. From the spectrum of $\mathrm{D}_{35}^{+}$(Figure 6a), it is apparent that all lighter members of this series lost the $w$-carbon of the alkyl chain. Discerning the mechanism of this new reaction will require further study.

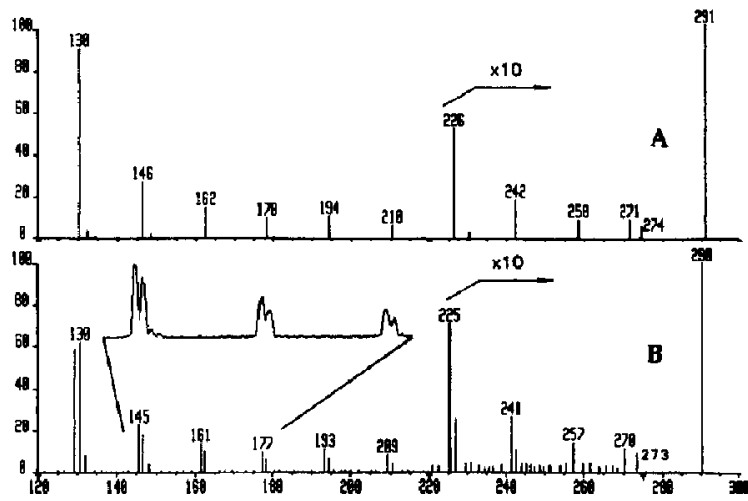

Figure 6. 400-eV Q-scan product ion spectra of (a) isotopically pure $D_{35}^{+}$and (b) the mislabeled $D_{34}^{+}$. The inset shows an expanded part of the original (MCA) data from which the mass/intensity pairs were calculated.
It is important to note that for analytes that do not isotopically differentiate the $N$-methyls from the long chain (e.g., $\mathrm{D}_{0}^{+}$or $D_{35}^{+}$), these two series become isomeric and indistinguishable. Therefore, those signals attributed above to CRF of $D_{0}^{+}$and $D_{35}^{+}$are in fact partially due to reactions proximate to the charge site. The generality of this alternative reaction is under investigation. It may apply only to tetraalkylammonium ions, and it may be limited to the experimental conditions used here $(400$ $\mathrm{eV}$ collision energy; multicollision with argon). It has been shown [22] that mild collision conditions $(30 \mathrm{eV}$; single collision) do not cause loss of $\mathrm{N}$-methyl groups from $\mathrm{D}_{29}^{+}$.

\section{Conclusions}

These studies established that both surfactants ( 2 and 3) have $>98 \%$ label purity at each supposedly labeled site and that the mislabeling is statistically distributed among those sites. The conclusion of random mislabeling of these materials remains firm despite the discovery of a new reaction competitive to CRF. However, if the mislabeling of 3 had been site-specific rather than random, the alternative reaction would have skewed the quantitative evaluation of the product ion spectrum of $\mathrm{D}_{34}^{+}$by giving the appearance of more random mislabeling at a site closer to the nitrogen. Better understanding of the relative importance of these reactions witl be needed to establish the utility of CRF in labeling studies.

\section{Acknowledgments}

This work was supported in part by grants from the National Science Foundation (no. DMR-84-06825, cofunded by the Army Research Office; no. CHE-88-22787; and no. CHE-86-11586). The University of Tennessee-Knoxville Chemistry Mass Spectrometry Center is funded by the Science Alliance, a State of Tennessee Center of Excellence. The National Science Foundation Chemical Instrumentation Program also contributed to the acquisition of the ZAB-EQ (grant no. CHE-86-09251).

\section{References}

1. Barber, M.; Bordoli, R. S.; Elliolt, G. J.; Sedgewick, R. D.; Tyler, A. N. Anal. Chem. 1982, 54, 645A-657A.

2. Jensen, N. J.; Tomer, K. B.; Gross, M. L.; Lyon, P. A. In Desorption Mass Spectromelry; Lyon, P. A., Ed.; American Chemical Society: Washington, DC, 1985; p. 194.

3. Tomer, K. B.; Jensen, N. J.; Gross, M. L. Anal. Chem. 1986, $58,2429-2433$.

4. Adams, J.; Gross, M. L. Org. Mass Spectrom. 1988, 23, 307-316.

5. Tomer, K. B.; Crow, F. W.; Gross, M. L. J. Am. Chem. Soc. 1983, $105,5487-5488$.

6. Jensen, N. J.; Tomer, K. B.; Gross, M. L. Anal. Chem. 1985 , 57, 2018-2021.

7. Adams, J.; Gross M. L. Anal. Chem. 1987, 59, 1576-1582.

8. Jensen, N. J.; Gross, M. L. Lipids 1986, 21, 362-365.

9. Jensen, N. J.; Tomer, K. B.; Gross, M. L. T. Am. Chem. Soc, 1985, 107. 1863-1868. 
10. Scott, A. B.; Tartar, H. V. J. Am. Chem. Soc. 1943, 65, 692-698.

11. Mysels, K. J.; Mukerjee, P. Critical Micelle Concentrations of Aqueous Surfactant Systems; NSRDS-NBS 36; U.S. Department of Commerce: Washingtan, DC, 1971.

12. Jennings, K. R.; Mason, R. S. In Tandem Mass Spectrometry; McLafferty, F. W., Ed.; Wiley: New York, 1983; p 197.

13. Crow, F. W.; Tomer, K. B.; Gross, M. L. Mass Spectrom. Rev. 1983, 2, 47-76.

14. Adams, J.; Gross, M. L. I. Am. Chem. Soc, 1986, 108, 6915-6921.

15. Deterding L. J.; Gross, M. L. Org. Mass Spectrom. 1988, 23, 169-177.

16. Cody, R. B. Rapid Commun. Mass Spectrom. 1988, 2, 260-261.
17. Yost, R. A.; Enke, C. G. In Tandem Mass Spectrometry; McLafferty, F. W., Ed; Wiley: New York, 1983; p 175.

18. Wysocki, V. H.; Bier, M. E.; Cooks, R. G. Org. Mass Spectrom. 1988, 23, 627-633.

19. Wysocki, V. H.; Ross, M. M.; Horning, S. R.; Cooks, R. G. Raprid Commun. Mass Spectrom. 1988, 2, 214217.

20. Fraley, D. F.; Lawrence, D. L. 33rd Annual Conference on Mass Spectrometry and Allied Topics, San Diego, May 26-31, 1985; pp 144-145.

21. Gierlich, H. H.; Röllgen, F. W.; Borchers, F.; Levsen, K. Org. Mass Spectrom. 1977, 12, 387-390.

22. Tuinman, A. A.; Cook, K. D. Rapid Commun. Mass Spectrom. 1989, 3, 289-292. 